\title{
COMPARISON BETWEEN PERFUSION COMPUTED TOMOGRAPHY AND DYNAMIC CONTRAST- ENHANCED MAGNETIC RESONANCE IMAGING IN RECTAL CANCER
}

Citation for published version (APA):

Kierkels, R. G. J., Backes, W. H., Janssen, M. H. M., Buijsen, J., Beets-Tan, R. G. H., Lambin, P., Lammerng, G., Oellers, M. C., \& Aerts, H. J. W. L. (2010). COMPARISON BETWEEN PERFÚSION COMPUTED TOMOGRAPHY AND DYNAMIC CONTRAST-ENHANCED MAGNETIC RESONANCE IMAGING IN RECTAL CANCER. International Journal of Radiation Oncology Biology Physics, 77(2), 400408. https://doi.org/10.1016/j.ijrobp.2009.05.015

Document status and date:

Published: 01/06/2010

DOI:

10.1016/j.jijrobp.2009.05.015

Document Version:

Publisher's PDF, also known as Version of record

\section{Document license:}

Taverne

\section{Please check the document version of this publication:}

- A submitted manuscript is the version of the article upon submission and before peer-review. There can be important differences between the submitted version and the official published version of record. People interested in the research are advised to contact the author for the final version of the publication, or visit the DOI to the publisher's website.

- The final author version and the galley proof are versions of the publication after peer review.

- The final published version features the final layout of the paper including the volume, issue and page numbers.

Link to publication

\footnotetext{
General rights rights.

- You may freely distribute the URL identifying the publication in the public portal. please follow below link for the End User Agreement:

www.umlib.nl/taverne-license

Take down policy

If you believe that this document breaches copyright please contact us at:

repository@maastrichtuniversity.nl

providing details and we will investigate your claim.
}

Copyright and moral rights for the publications made accessible in the public portal are retained by the authors and/or other copyright owners and it is a condition of accessing publications that users recognise and abide by the legal requirements associated with these

- Users may download and print one copy of any publication from the public portal for the purpose of private study or research.

- You may not further distribute the material or use it for any profit-making activity or commercial gain

If the publication is distributed under the terms of Article 25fa of the Dutch Copyright Act, indicated by the "Taverne" license above, 


\title{
COMPARISON BETWEEN PERFUSION COMPUTED TOMOGRAPHY AND DYNAMIC CONTRAST-ENHANCED MAGNETIC RESONANCE IMAGING IN RECTAL CANCER
}

\author{
Roel G. J. Kierkels, M.Sc., ${ }^{*}$ Walter H. Backes, M.Sc., Ph.D., ${ }^{\dagger}$ Marco H. M. Janssen, M.Sc., ${ }^{*}$ \\ Jeroen Buijsen, M.D., ${ }^{*}$ Regina G. H. Beets-Tan, M.D., Ph.D., ${ }^{\dagger}$ Philippe Lambin, M.D., Ph.D., ${ }^{*}$ \\ Guido Lammering, M.D., Ph.D., ${ }^{*}$ Michel C. Oellers, M.Sc., Ph.D., ${ }^{*}$ \\ and Hugo J. W. L. Aerts, M.Sc.* \\ * Department of Radiation Oncology and ${ }^{\dagger}$ Department of Radiology, Maastricht University Medical Center, GROW School for \\ Oncology and Developmental Biology, Maastricht, The Netherlands
}

\begin{abstract}
Purpose: To compare pretreatment scans with perfusion computed tomography (pCT) vs. dynamic contrastenhanced magnetic resonance imaging (DCE-MRI) in rectal tumors.

Methods and Materials: Nineteen patients diagnosed with rectal cancer were included in this prospective study. All patients underwent both pCT and DCE-MRI. Imaging was performed on a dedicated 40-slice CT-positron emission tomography system and a 3-T MRI system. Dynamic contrast enhancement was measured in tumor tissue and the external iliac artery. Tumor perfusion was quantified in terms of pharmacokinetic parameters: transfer constant $K^{\text {trans }}$, fractional extravascular-extracellular space $v_{e}$, and fractional plasma volume $v_{p}$. Pharmacokinetic parameter values and their heterogeneity (by $80 \%$ quantile value) were compared between pCT and DCE-MRI. Results: Tumor $K^{\text {trans }}$ values correlated significantly for the voxel-by-voxel-derived median (Kendall's $\tau$ correlation, $\tau=0.81, p<0.001)$ and $80 \%$ quantile $(\tau=0.54, p=0.04)$, as well as for the averaged uptake $(\tau=0.58, p=0.03)$. However, no significant correlations were found for $v_{e}$ and $v_{p}$ derived from the voxel-byvoxel-derived median and $80 \%$ quantile and derived from the averaged uptake curves.

Conclusions: This study demonstrated for the first time that pCT provides $K^{\text {trans }}$ values comparable to those of DCE-MRI. However, no correlation was found for the $v_{e}$ and $v_{p}$ parameters between CT and MRI. Computed tomography can serve as an alternative modality to MRI for the in vivo evaluation of tumor angiogenesis in terms of the transfer constant $K^{\text {trans }}$. (C) 2010 Elsevier Inc.
\end{abstract}

Perfusion CT, DCE-MRI, Pharmacokinetic model, Rectal cancer, Tumor perfusion.

\section{INTRODUCTION}

Perfusion computed tomography (pCT) and dynamic contrast-enhanced magnetic resonance imaging (DCE-MRI) are clinical imaging techniques that are increasingly applied to noninvasively assess the microvascular status of tumor tissue (1-3). In clinical cancer research, regression of tumor microvasculature is considered an important early surrogate marker for treatment response, before reductions in tumor volume become apparent. To date, both pCT and DCEMRI are increasingly used for the prediction and evaluation of treatment response (4-6), as indicators of tumor angiogenesis (7-9), and for primary tumor staging (10). Computed tomography has the advantage of generally being more easily accessible compared with MRI. Moreover, the majority of patients with solid tumors receive radiotherapy for which CT or CT-positron emission tomography (PET) examinations are applied. Therefore, the use of pCT in the assessment of tumor microcirculation could lead to important logistical advantages.

Both pCT and DCE-MRI are imaging modalities that rely on the dynamic assessment of tracer uptake kinetics, subsequently quantified by means of pharmacokinetic models (11). These models describe, in terms of pharmacokinetic parameters, the wash-in and wash-out of contrast agent from the microvasculature into the surrounding tissues. A commonly applied pharmacokinetic two-compartment model is the extended Kety model, which consists of the (transendothelial) volume transfer constant $K^{\text {trans }}$ (in $\min ^{-1}$ ), the fractional
Reprint requests to: Hugo J. W. L. Aerts, M.Sc., Department of Radiation Oncology (MAASTRO), Maastricht University, Dr. Tanslaan 12, 6202 AZ Maastricht, The Netherlands. Tel: (+31) (0) 88-44-55-666; Fax: (+31) (0) 88-44-55-667; E-mail: Hugo.Aerts@maastro.nl

Supported by the Netherlands Organization for Health Research and Development (clinical fellowship awarded to G.L.). Technical and financial support provided by Siemens.

G.L., M.C.O., and H.J.W.L.A. contributed equally to this article. Conflict of interest: none.

Acknowledgment-The authors thank Henk Schoenmakers for coordination of the MRI scans.

Received Feb 11, 2009, and in revised form May 19, 2009. Accepted for publication May 19, 2009. 
volume of the extravascular-extracellular space (EES) $v_{e}$, and the fractional blood plasma volume $v_{p}$. Clinically, $K^{\text {trans }}$ is the most valuable pharmacokinetic parameter, which describes the transfer rate of the contrast agent from the plasma space to the EES, and represents a combination of microvascular blood flow, vessel wall permeability, and vessel density (i.e., permeability surface area product) (11).

Although the principles of pCT and DCE-MRI are similar, the physical principles, imaging protocols, pharmacokinetic properties of contrast agents, and injection protocols strongly differ. Because contrast enhancement not only depends on the microvasculature of the tissue under study but also on the imaging method, a priori it is unknown to what extent different imaging modalities provide comparable quantitative information on the tumor microcirculation, in terms of reliable pharmacokinetic parameter estimates.

The objective of this study was to compare the pharmacokinetic parameters characterizing tumor microcirculation, derived from pretreatment $\mathrm{pCT}$ and DCE-MRI scans in rectal cancer patients.

\section{METHODS AND MATERIALS}

\section{Patient characteristics}

From November 2007 to September 2008, a total of 19 patients (15 men and 4 women, aged $64.3 \pm 7.2$ years) were included. All patients were diagnosed with non-locally advanced primary resectable rectal cancer, Stage I-III according to International Union Against Cancer criteria. After the pCT and DCE-MRI examination, all patients were treated with 5 fractions of 5 Gy radiotherapy on 5 consecutive days, which was followed by total mesorectal excision within 3 days of the last radiotherapy fraction. The local medical ethics committee approved the trial, according to Dutch law. Patients gave written informed consent before inclusion.

\section{CT-PET and $p C T$}

The CT-PET and pCT examinations were performed on a dedicated Siemens TruePoint Biograph 40 PET-CT simulator (Siemens Medical, Erlangen, Germany). A static CT-PET scan was performed for tumor detection, delineation, and radiotherapy treatment planning. For the PET scan, an intravenous injection of (weight $[\mathrm{kg}]$ $\times 4+20) \mathrm{MBq}$ fluorodeoxyglucose (FDG) (GE Healthcare, Eindhoven, The Netherlands) was performed, followed by $10 \mathrm{~mL}$ saline. After a 60-min uptake period, CT-PET images were acquired. The static CT scan was performed without iodine contrast. For the PET scan, three bed positions, each of $5 \mathrm{~min}$, were acquired. Computed tomography-based attenuation, three-dimensional scatter correction, and decay correction were performed during reconstruction of the PET scan.

In addition to the routine CT-PET protocol, a pCT was performed. The scanning range for the perfusion scan was defined by an expert radiation oncologist (J.B. or G.L). The pCT scan was performed using an iodinated contrast agent with a concentration of $300 \mathrm{mg}$ iodine $/ \mathrm{mL}$ (Xenetix 300; Guerbet, Aulnay-sous-Bois, France). A volume of $60 \mathrm{~mL}(n=13)$ and $120 \mathrm{~mL}(n=6)$ of contrast agent was injected at a rate of $3 \mathrm{~mL} / \mathrm{s}$ with an automatic injector (Stellant CT Injection System; MedRad, Warrendale, PA) into the antecubital fossa. It was found that the vascular input functions were abnormally shaped (possibly reflecting multiple recirculations) on our CT system for 120 -mL injections. Therefore, we adapted the protocol during the study to $60 \mathrm{~mL}$, which yielded better results. The pCT scan was performed in static cine mode and contained 12 contiguous slices with a reconstructed slice thickness of $2.4 \mathrm{~mm}$, a field of view (FOV) of $500 \mathrm{~mm}$, and an image size of $512 \times 512$. Other acquisition settings were as follows: tube voltage $80 \mathrm{kVp}$, tube current $140 \mathrm{mAs}$, rotation time $1 \mathrm{~s}$, and total acquisition time $100 \mathrm{~s}$.

\section{DCE-MRI}

Dynamic contrast-enhanced MRI was performed on a clinical 3-T MRI system (Achieva; Philips Medical Systems, Best, The Netherlands) equipped with a five-element cardiac phased-array coil. Sagittal and transverse T2-weighted anatomic images of the abdominal and tumor region were acquired according to the location of the previously acquired CT images. To obtain MR slices in the same orientation as the CT images, all MR slices were angulated perpendicular to the table. To determine the precontrast $\mathrm{T} 1$ relaxation time of the tumor and gluteus muscle, nine T1-weighted scans (three-dimensional fast gradient recalled gradient echo) were acquired with different flip angles $\left(1^{\circ}, 2^{\circ}, 3^{\circ}, 5^{\circ}, 7^{\circ}, 10^{\circ}, 15^{\circ}, 25^{\circ}\right.$, and $\left.35^{\circ}\right)$, repetition time $11 \mathrm{~ms}$, echo time $4.6 \mathrm{~ms}, 10$ transverse slices, slice thickness $8 \mathrm{~mm}$, matrix dimension $128 \times 128$, and pixel size $2 \times 2 \mathrm{~mm}$. The T1-weighted dynamic contrast-enhancement series used the same sequence with a flip angle of $35^{\circ}$ and comprised 125 volume acquisitions (acquisition time, $490 \mathrm{~s}$ ) at a scan interval of $3.92 \mathrm{~s}$. At the start of the acquisition of the 11th dynamic volume, the injection of the contrast agent was initiated. Gadopentetate dimeglumine $(0.2 \mathrm{mmol} / \mathrm{kg}$ body weight, $0.5 \mathrm{mmol} / \mathrm{mL}$; Magnevist; Schering, Berlin, Germany) was injected in the antecubital fossa at a rate of $1 \mathrm{~mL} / \mathrm{s}$ and flushed with $25 \mathrm{~mL}$ saline. This relatively low injection rate consistently provided bolus-shaped vascular input functions at $3 \mathrm{~T}$. Higher injection rates gave rise to strong degradations of the bolus peak due to the $\mathrm{T} 2 *$ and susceptibility effects, which was found in initial test acquisitions during the setup of the DCE-MRI protocol (data not shown). For comparison, pCT and DCE-MRI protocols are summarized in Table 1.

\section{Pharmacokinetic analysis}

Image coregistration. Automatic image registration between the precontrast CT, pCT, and PET images was performed using an algorithm based on mutual information (Focal software, version 4.34; CMS, St. Louis, MO) (12). For every patient, the tumor was delineated according to automatic standardized uptake value thresholding of the PET data using the TrueD system (VC50; Siemens Medical, Erlangen, Germany) $(13,14)$. The tumor delineations were projected on the registered pCT. To ensure correct matching, the tumors on the T2-weighted MR images were delineated by a radiation oncologist specializing in rectal cancer (J.B.), with the knowledge of all MR, $\mathrm{CT}$, and PET image information. All voxels within tumor delineations on pCT and DCE-MRI were used for further pharmacokinetic parameter analysis. All voxels within tumor delineations on pCT and DCE-MRI were used for further pharmacokinetic parameter analysis.

Data analysis. The acquired pCT data were downsampled from $0.98 \times 0.98 \times 2.4-\mathrm{mm}$ to $3.92 \times 3.92 \times 4.8-\mathrm{mm}$ voxels to improve the signal-to-noise ratio (SNR). For both pCT and DCE-MRI the relative signal change was derived by dividing the measured signal-enhancement curves by the signal intensity acquired from the precontrast baseline images. For pCT, the intensity values were rescaled such that the intensity in air was zero, to ensure that the $\mathrm{CT}$ value was proportional to the attenuation 
Table 1. Overview of the application of pCT and DCE-MRI acquisition parameters and contrast agents

\begin{tabular}{lcc}
\hline \multicolumn{1}{c}{ Parameter } & pCT & DCE-MRI \\
\hline Acquisition time (s) & 100 & 490 \\
Interscan interval (s) & 1.00 & 3.92 \\
Reconstructed pixel size (mm) & $3.92 \times 3.92^{*}$ & $2 \times 2$ \\
Slice thickness (mm) & $4.80^{*}$ & 8 \\
Field-of-view (LR/AP/FH) (mm) & $500 \times 500 \times 28.8$ & $256 \times 256 \times 80$ \\
Contrast agent (CA) & Iobitridol $(300 \mathrm{mg} / \mathrm{mL})$ & Gadopentetate dimeglumine $(0.5 \mathrm{mmol} / \mathrm{mL})$ \\
CA molecular weight $(\mathrm{g} / \mathrm{mol})$ & 835 & 938 \\
CA viscosity $(\mathrm{mPa} \cdot \mathrm{s})$ at $37^{\circ} \mathrm{C}$ & 6.0 & 2.0 \\
Injection volume $(\mathrm{mL})$ & 60 or 120 & 30 \\
Injection rate $(\mathrm{mL} / \mathrm{s})$ & 3 & 1 \\
\hline
\end{tabular}

Abbreviations: $\mathrm{pCT}=$ perfusion computed tomography; DCE-MRI = dynamic contrast-enhanced magnetic resonance imgaging; $\mathrm{LR}=$ left right, $\mathrm{AP}=$ anterior-posterior; $\mathrm{FH}=$ feet-head.

* Downsampled for pharmacokinetic analysis from reconstructed CT data.

coefficient and the contrast agent concentration. To correct for the nonlinear relationship for MRI, the T1-weighted signal amplification was fitted to the Ernst equation (15). The T1 relaxation time before contrast agent injection (T10) was set to $1664 \mathrm{~ms}$ for arterial blood at $3 \mathrm{~T}(16)$.

Pharmacokinetic model. For the quantification of the pCT and DCE-MRI time series data, the extended Kety two-compartment model was used (11), describing the tissue uptake of a contrast agent from the blood plasma into the tissue by:

$$
C_{t}(t)=v_{p} C_{p}(t)+K^{\text {trans }} \int_{0}^{t} c_{p}(u) e^{\frac{-k_{e} \text { trans }}{v_{e}}(t-u)} d u
$$

The blood plasma concentration curve $\left(C_{p}\right)$ was derived from the acquired whole-blood tracer concentration $\left(C_{b}\right)$ divided by (1-Hct) (17), with Hct $=0.45$ the hematocrit value (11). For both DCEMRI and pCT the blood concentration was extracted from the right external iliac artery. To improve the SNR, $C_{p}$ was calculated by averaging the concentration time curves over all voxels selected inside the iliac artery. The tumor tissue concentration curves $\left(C_{t}\right)$ were acquired from the time series data and extracted on a voxelby-voxel basis and on a tumor uptake curve-based average from all tumor voxels. Pharmacokinetic data analysis was performed using in-house-developed software in MATLAB (Mathworks, Natick, MA). The concentration time curves from pCT and DCEMRI data were fitted to the pharmacokinetic model. Subsequently, pharmacokinetic parameters were calculated using the LevenbergMarquardt algorithm (using the "lsqcurvefit" routine). The parameter boundaries were set to $0 \leq K^{\text {trans }} \leq 5 \mathrm{~min}^{-1}, 0 \leq v_{e} \leq 1$, and $0 \leq v_{p} \leq 1(18-20)$.

\section{Statistical analysis}

All results were expressed as mean \pm standard deviation (SD). The distributions of the voxel-by-voxel-derived pharmacokinetic parameter values were compared by histogram
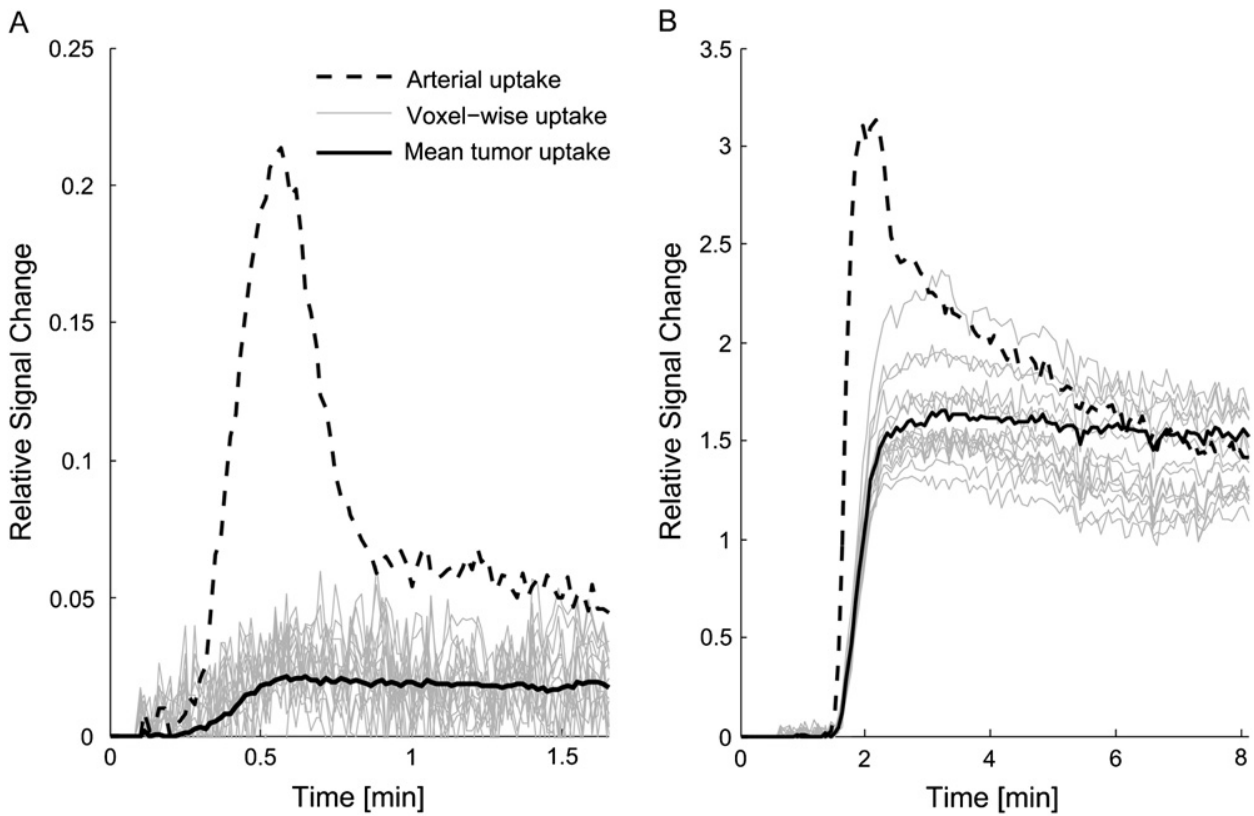

Fig. 1. Contrast agent uptake curves in arterial and tumor tissue of a representative patient, resulting from perfusion computed tomography (A) and dynamic contrast-enhanced magnetic resonance imaging (DCE-MRI) (B). The voxel-by-voxel curves (gray) and a tumor average uptake curve (black) of the tumor tissue are shown. Note the differences of the time axes. The signal-to-noise ratio of the DCE-MRI was superior in the tumor tissue, for both the voxel-by-voxel and tumor average uptake curve. 

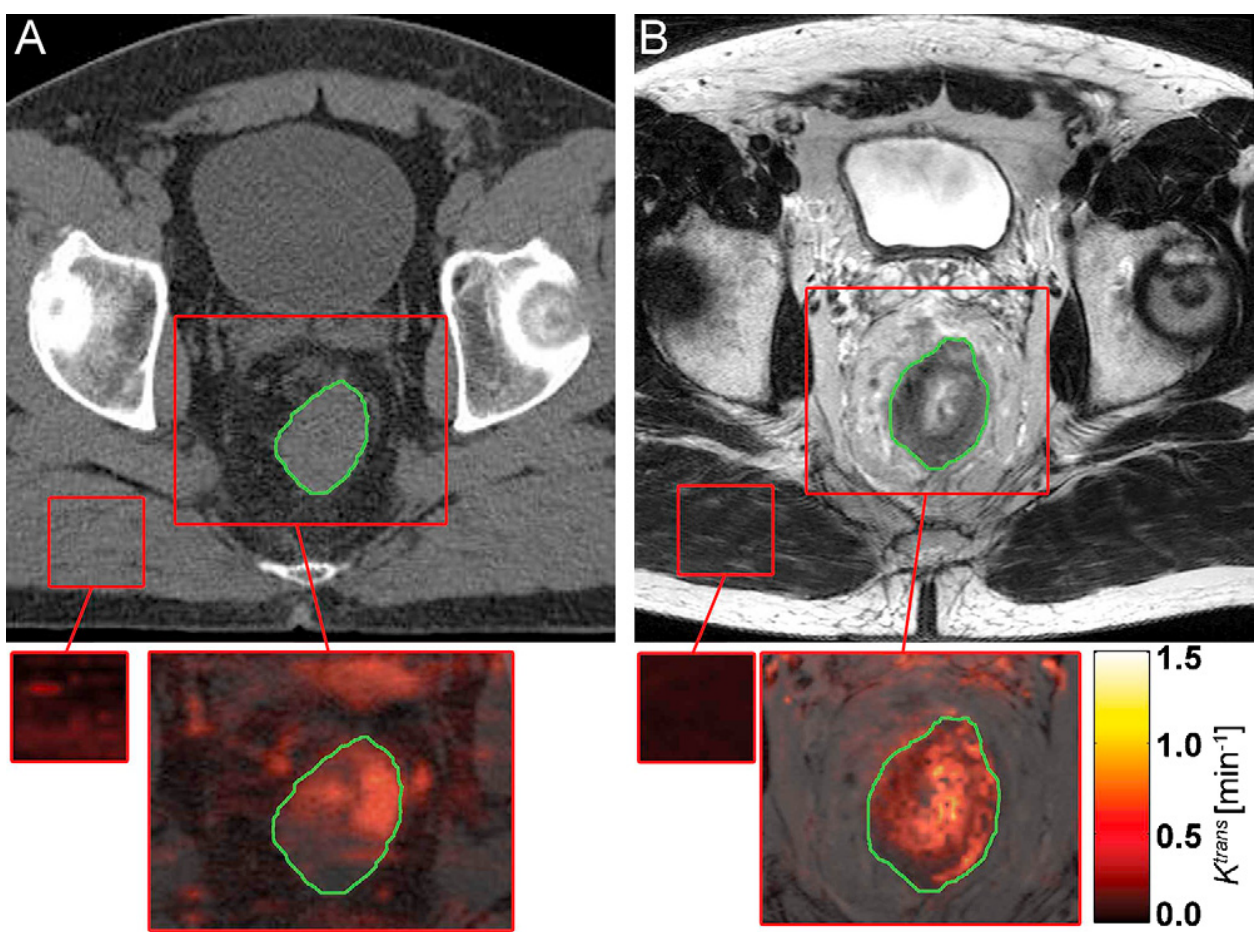

Fig. 2. Comparison between computed tomography (A) and a T2-weighted magnetic resonance imaging (B) of a representative rectum cancer patient. The upper row displays the anatomic precontrast images, the lower row the $K^{\text {trans }}$ maps of the muscle and tumor tissue regions, as indicated by the boxes in the anatomic images. Note that hyperintensities $\left(i . e\right.$., $K^{\text {trans }}$ $\geq 1 \mathrm{~min}^{-1}$ ) in the $K^{\text {trans }}$ maps can only be observed in the tumor and not the gluteus muscle region.

analysis and characterized by the corresponding median and the $80 \%$ quantile value of the histograms. Statistical correlations between estimates were evaluated using the Kendall's $\tau$ correlation coefficient and Bland-Altman plots. Differences were considered to be statistically significant when $p<0.05$. The differences between volume of interest (VOI)-based and voxel-based (i.e., median and $80 \%$ quantile) values were tested using the Wilcoxon signed-rank test.

\section{RESULTS}

\section{Patient characteristics}

To compare pharmacokinetic parameters, individual pCT and DCE-MRI scans were analyzed for every patient. In 15 of 19 patients, images suitable for comparative pharmacokinetic analysis were obtained. Four patients were excluded owing to contrast agent leakage during injection or strong
A

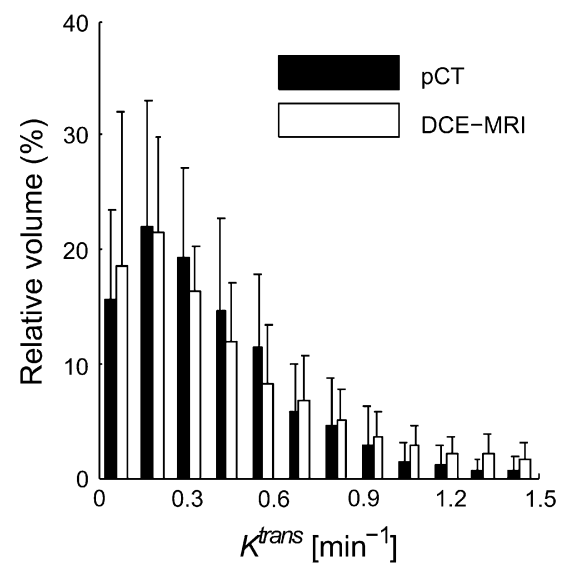

B

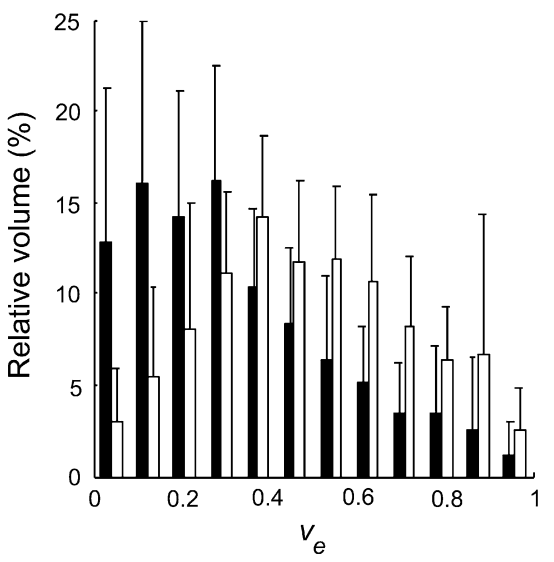

C

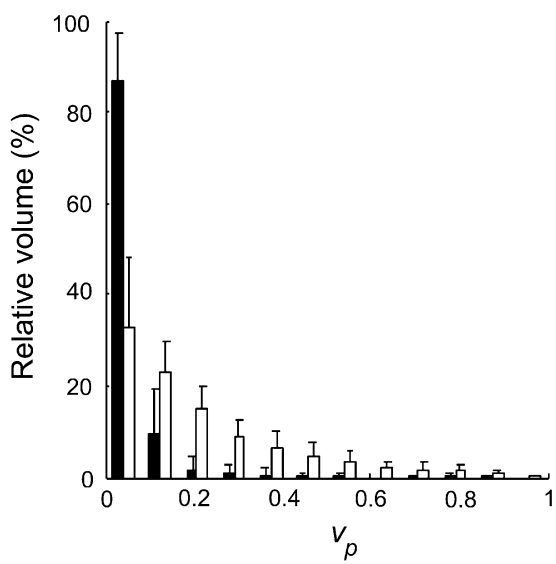

Fig. 3. Histograms of the pharmacokinetic-parameters $K^{\text {trans }}(\mathrm{A}), v_{e}(\mathrm{~B})$, and $v_{p}(\mathrm{C})$ of the tumor tissue derived from the voxel-by-voxel analysis of perfusion computed tomography (pCT) and dynamic contrast-enhanced magnetic resonance imaging (DCE-MRI) scans. The data are shown relative to the analyzed tumor volume. Error bars denote the standard deviation between patients. Note the similarities of the $K^{\text {trans }}$ distribution for both imaging modalities and the higher DCE-MRI-derived $K^{\text {trans }}$ values at increasing $K^{\text {trans }}\left(>0.6 \mathrm{~min}^{-1}\right)$. 


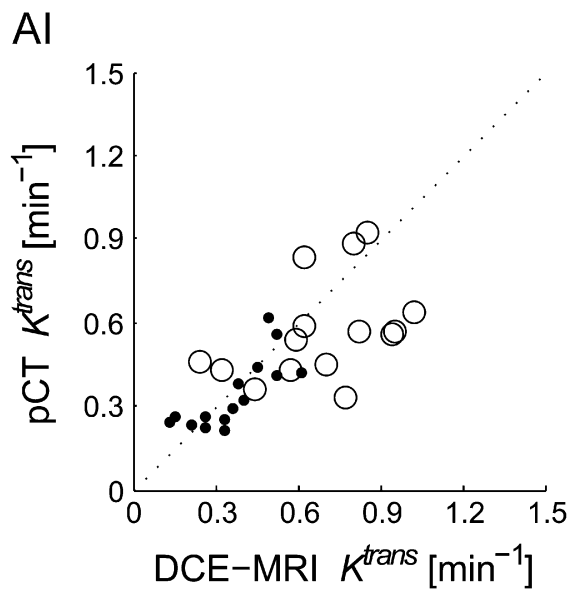

$\mathrm{BI}$

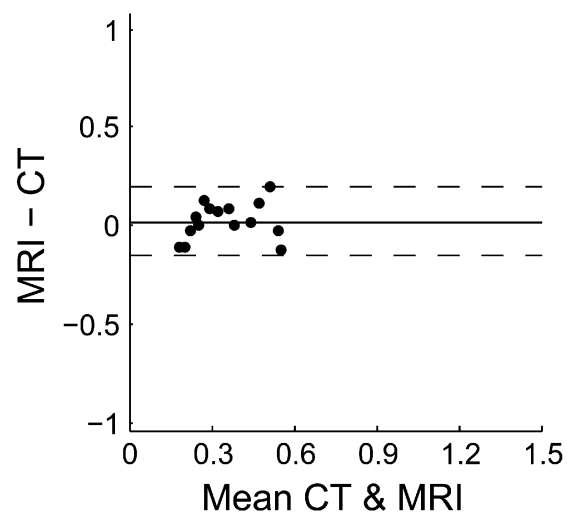

All

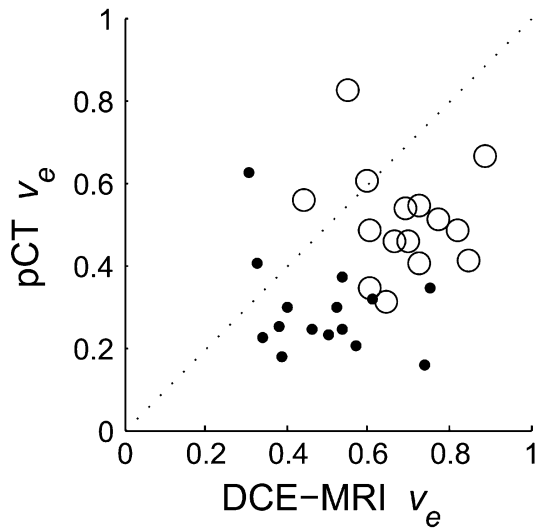

BII

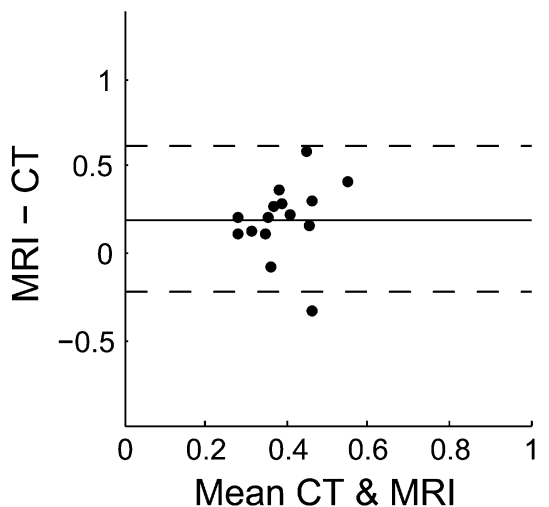

Alll

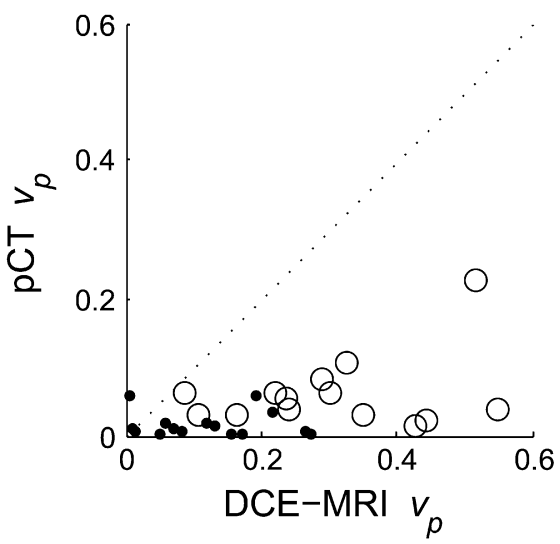

BIII

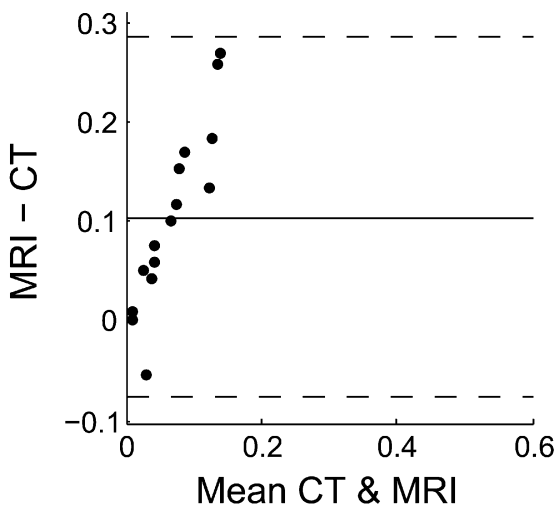

Fig. 4. Scatter plots (A) and Bland-Altman plots (B) of the median (dots) and the $80 \%$ quantile (circles) of the tumor pharmacokinetic parameter $K^{\text {trans }}$ (Panel I), $v_{e}$ (Panel II), and $v_{p}$ (Panel III) of pCT versus DCE-MRI. Parameters were obtained on the basis of a voxel-by-voxel pharmacokinetic analysis. The dotted line (A) represents perfect agreement between perfusion computed tomography (pCT) and dynamic contrast-enhanced magnetic resonance imaging (DCE-MRI)-derived parameters. The solid lines and dashed lines (B) indicate the mean difference and the $95 \%$ (1.96 SD) quantile limits of the parameters derived from the two imaging modalities. The corresponding correlation coefficients and statistics are listed in Table 2.

abdominal motion during pCT acquisition. All patients underwent the DCE-MRI examination after the pCT examination $(1.61 \pm 1.13$ days). The total tumor volume (based on the static CT-PET data) was $15.6 \pm 15.2 \mathrm{~cm}^{3}$.

\section{Contrast enhancement}

Figure 1 illustrates the contrast uptake curves for pCT and DCE-MRI of a representative patient. Because of the longer acquisition time, with MRI it is possible to measure the late tracer kinetics in the artery and tumor tissue. The SNR of the voxel-by-voxel tumor tissue curves was significantly higher for DCE-MRI $(20.1 \pm 6.4)$ compared with pCT $(6.7 \pm 5.3)(p<0.01)$. In addition, the SNR of the mean tumor uptake curves was significantly higher for DCE-MRI $(62.4 \pm 19.9)$ than for pCT $(23.6 \pm 25.0)(p<0.01)$.

\section{Comparison images between $p C T$ and DCE-MRI}

An example of a voxel-by-voxel parameter map of $K^{\text {trans }}$ for $\mathrm{pCT}$ and DCE-MRI is depicted in Fig. 2. Apparently there is a visual agreement between the parameter maps. However, some differences possibly introduced by differences in patient position and bladder and rectum filling were observed. For both imaging modalities it is evident that the spatial distribution of $K^{\text {trans }}$ in healthy muscle tissue is relatively homogeneous and values are low compared with tumor tissue, in which $K^{\text {trans }}$ is more heterogeneously distributed with local high- $K^{\text {trans }}$ regions.

\section{Voxel-by-voxel parameters}

The histograms of the voxel-by-voxel-determined pharmacokinetic parameter estimates are shown in Fig. 3. Similarities between the distributions of the pCT- and the DCE-MRI-derived $K^{\text {trans }}$ values were observed (Fig. 3A). However, DCE-MRI includes more high $K^{\text {trans }}$ values $\left(>0.6 \mathrm{~min}^{-1}\right)$. Overall, for $v_{e}$ higher values for DCE-MRI were observed compared with pCT (Fig. 3B). The same was observed for the distribution of $v_{p}$ (Fig. 3C).

The relationships between the voxel-by-voxel-derived median and $80 \%$ quantile pharmacokinetic parameter values derived from DCE-MRI and pCT are shown in Fig. 4. Both the median $K^{\text {trans }}(\tau=0.81, p<0.001)$ and the $80 \%$ quantile $K^{\text {trans }}(\tau=0.54, p=0.04)$ were significantly correlated 
Table 2. Comparison of pharmacokinetic parameter values between pCT and DCE-MRI

\begin{tabular}{|c|c|c|c|c|}
\hline \multirow[b]{2}{*}{ Parameter } & \multirow{2}{*}{$\begin{array}{c}\text { (VOI analysis) } \\
\text { Tumor }(\text { mean } \pm \text { SD) }\end{array}$} & \multicolumn{2}{|c|}{ (Voxel analysis) tumor } & \multirow{2}{*}{$\begin{array}{c}\text { (Voxel analysis) } \\
\text { Muscle (median } \pm \text { SD) }\end{array}$} \\
\hline & & Median \pm SD & $80 \%$ quantile $\pm \mathrm{SD}$ & \\
\hline \multicolumn{5}{|l|}{$K^{\text {trans }}\left(\min ^{-1}\right)$} \\
\hline $\mathrm{pCT}$ & $0.30 \pm 0.16$ & $0.34 \pm 0.13$ & $0.57 \pm 0.18$ & $0.08 \pm 0.05$ \\
\hline DCE-MRI & $0.53 \pm 0.37$ & $0.37 \pm 0.14$ & $0.69 \pm 0.23$ & $0.13 \pm 0.08$ \\
\hline \multicolumn{5}{|l|}{$v_{e}$} \\
\hline $\mathrm{pCT}$ & $0.31 \pm 0.25$ & $0.29 \pm 0.12$ & $0.51 \pm 0.13$ & $0.26 \pm 0.13$ \\
\hline DCE-MRI & $0.66 \pm 0.25$ & $0.49 \pm 0.14$ & $0.69 \pm 0.12$ & $0.24 \pm 0.10$ \\
\hline Difference & $0.35 \pm 0.44(p=0.01)$ & $0.20 \pm 0.21(p=0.005)$ & $0.22 \pm 0.21(p=0.007)$ & $0.03 \pm 0.18(p=0.4)$ \\
\hline Kendall's $\tau$ & $-0.35(p=0.2)$ & $-0.19(p=0.5)$ & $-0.17(p>0.5)$ & $-0.24(p=0.4)$ \\
\hline \multicolumn{5}{|l|}{$v_{p}$} \\
\hline Kendall's $\tau$ & $0.23(p=0.4)$ & $-0.20(p=0.5)$ & $0.02(p=0.5)$ & $0.15(p=0.5)$ \\
\hline
\end{tabular}

Abbreviations: VOI = volume of interest $K^{\text {trans }}=($ transendothelial $)$ volume transfer constant; $v_{e}=$ volume of extravascular-extracellular space per unit volume of tissue; $v_{p}=$ blood plasma volume per unit volume of tissue; $\tau=$ Kendall's correlation coefficient. Other abbreviations as in Table 1.

(Fig. 4AI). No significant correlations were found for the $v_{e^{-}}$ derived median $(p=0.5)$ and $80 \%$ quantile $(p=0.5)$ (Fig. 4AII). Additionally, the $v_{p}$-derived median $(p=0.5)$ and $80 \%$ quantile $(p=0.9)$ were not significantly correlated (Fig. 4AIII). Table 2 lists all averaged pharmacokinetic parameters and statistical results.

The Bland-Altman plots in Fig. 4B demonstrate no significant differences between the medians and $80 \%$ quantile values of $K^{\text {trans }}$ ( $p=0.4$ and $p=0.09$, respectively) (Fig. 4BI). The pCT-derived median and $80 \%$ quantile $v_{e}$ values were both significantly lower than those of DCEMRI ( $p=0.005$ and $p=0.007$, respectively) (Fig. 4BII). Additionally, the derived median and $80 \%$ quantile $v_{p}$ values were both significantly lower for pCT $(p=0.001$ and $p<0.001$, respectively) (Fig. 4BIII).

For gluteus muscle tissue, the pCT-derived $K^{\text {trans }}$ was significantly lower $\left(0.08 \pm 0.05 \mathrm{~min}^{-1}\right)$ than that of DCE-MRI $\left(0.13 \pm 0.08 \mathrm{~min}^{-1}, p=0.003\right)$ (Table 2). Values for $v_{e}$ and $v_{p}$ in muscle, however, were not significantly different between the two modalities ( $p=0.4$ and $p=0.1$, respectively).

\section{Average tumor curve parameters}

The pharmacokinetic parameters of the average tumor contrast uptake curves are shown in Fig. 5. $K^{\text {trans }}$ values correlated significantly between $\mathrm{pCT}$ and DCE-MRI $(\tau=0.58$, $p=0.03)$. However, the $v_{e}$ and $v_{p}$ parameters did not significantly correlate ( $p=0.2$ and $p=0.4$, respectively). Note that for higher mean $K^{\text {trans }}$ the DCE-MRI-derived $K^{\text {trans }}$ increased more than for pCT (Fig. 5).

The Bland-Altman plots in Fig. 5B demonstrated significant differences between all pCT and DCE-MRI-derived parameters. Table 2 lists the averaged parameter estimates and statistical results. The $K^{\text {trans }}$ values were significantly lower for pCT $(p=0.002)$ (Fig. 5BI). Additionally, the $v_{e}$ and $v_{\mathrm{p}}$ were significantly lower for $\mathrm{pCT}(p=0.01$ and $p<0.01$, respectively) (Fig. 5BII and 5BIII).

\section{DISCUSSION}

To our knowledge, this is the first study performing (l) pCT and DCE-MRI in the same patients diagnosed with primary rectal cancer, and (2) DCE-MRI with pharmacokinetic modeling at $3 \mathrm{~T}$. Our study demonstrates that the transendothelial transfer constant $K^{\text {trans }}$ derived from pCT is significantly comparable to the one derived from DCE-MRI. Therefore, CT can serve as an alternative modality to MRI for the in vivo evaluation of tumor angiogenesis.

This study demonstrates that $\mathrm{pCT}$ allows for the noninvasive characterization of tumor perfusion, which can be easily performed at the moment when CT is used for radiotherapy treatment planning. Thus, pCT represents an attractive alternative to DCE-MRI in rectal cancer patients who are referred for radiotherapy. However, the pCT scan will expose the patient to additional ionizing radiation, whereas DCE-MRI does not involve any ionizing radiation exposure. In this regard, one has to compare this additional radiation to the effective dose of an FDG-PET scan (approximately $7 \mathrm{mSv}$ ), and particularly the fact that these patients will receive a much higher dose due to the scheduled radiotherapy. In addition, the additional dose of pCT has to be weighted against the relatively old age (frequently $>60$ years) and life expectancies of these patients. However, for relatively young patients and/or when MRI system availability is no limitation, DCE-MRI is recommended over pCT because of the better signal-to-noise characteristics, stronger contrast uptake, more complete evaluation of 

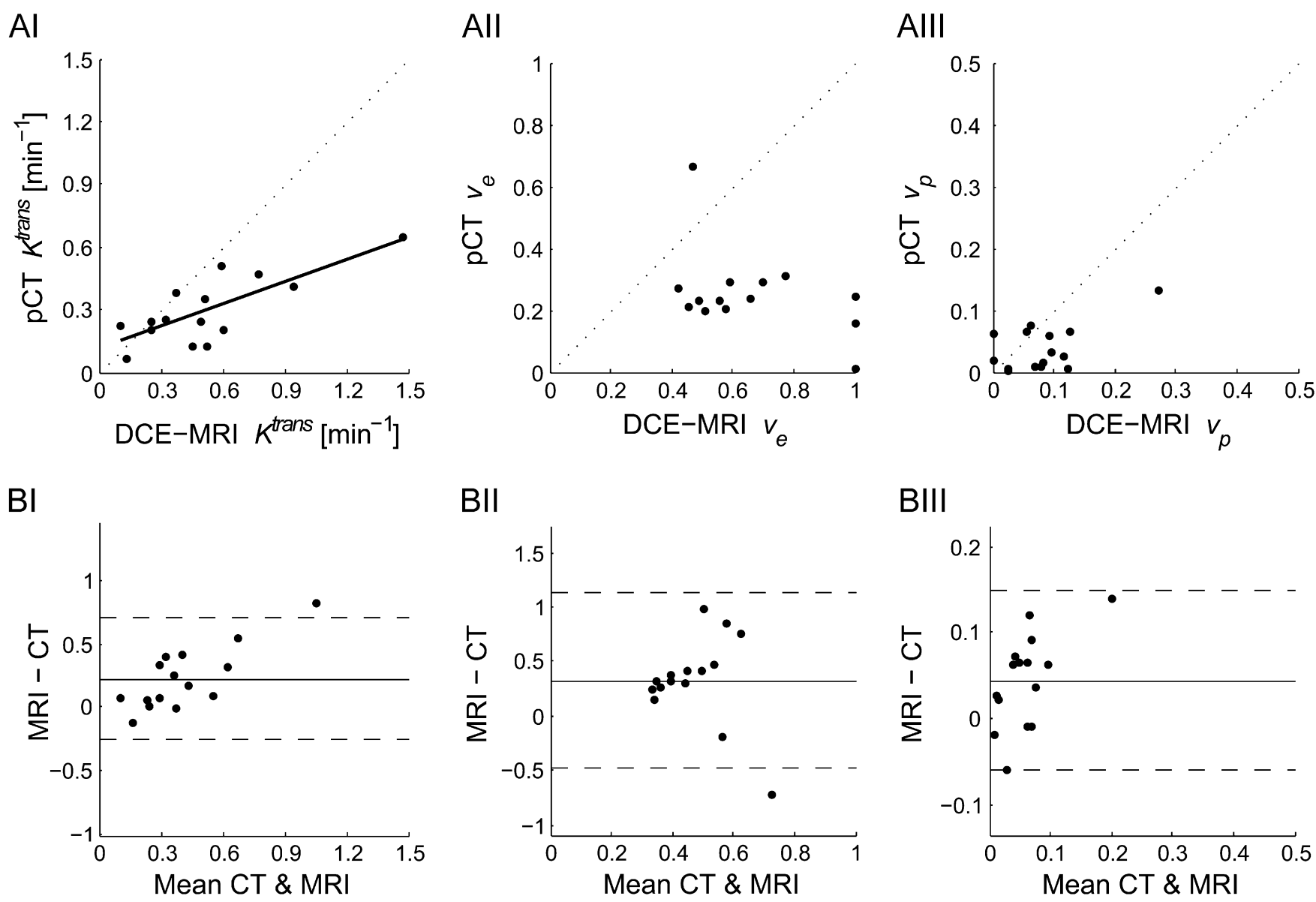

Fig. 5. Scatter plots (A) and Bland-Altman plots (B) of pharmacokinetic parameters $K^{\text {trans }}$ (Panel I), $v_{e}$ (Panel II), and $v_{p}$ (Panel III), derived from the mean tumor uptake curve of the perfusion computed tomography (pCT) and dynamic contrastenhanced magnetic resonance imaging (DCE-MRI) scans. Each dot represents the data of one patient. The dotted line (A) represents perfect agreement between pCT- and DCE-MRI-derived parameters. The black line indicates the results from the Kendall's $\tau$ correlation test for $K^{\text {trans }}$. In the Bland-Altman plot the solid line represents the mean of the difference between MRI and pCT, and the dashed lines indicate the 95\% (1.96 SD) confidence intervals. Corresponding statistics are listed in Table 2 .

the microcirculatory environment of the tumor, larger tumor coverage, and the absence of ionizing radiation. Qualifications of CT and MRI to perform perfusion imaging are summarized in Table 3.

The acquisition time of pCT (100 s) was long enough to assess measurements of tumor $K^{\text {trans }}$, which correlated to those of DCE-MRI (490 s) (21). In addition, the sampling time of pCT (1 s) and DCE-MRI (3.92 s) was expected to be sufficient for reliable estimation of $K^{\text {trans }}$ (22). Clinically, $K^{\text {trans }}$ is generally considered the most relevant perfusion parameter. However, the discrepancy for the $v_{e}$ and $v_{p}$ parameters might be explained by the fact that other factors (besides acquisition time and sample frequency) influence the measurements of the pharmacokinetic parameters-for example, differences in contrast agent, day-to-day physiologic variations (e.g., cardiac output), and signal-to-noise signal intensity characteristics. However, for $K^{\text {trans }}$ the used sampling and acquisition times of both pCT and DCE-MRI were sufficient. This was demonstrated by the fact that the voxel-by-voxel-based median and $80 \%$ quantile value and the volume-averaged time course-derived $K^{\text {trans }}$ were significantly correlated.
To date, only two studies have reported on a comparative study between pCT and DCE-MRI, unfortunately without pharmacokinetic modeling. One study assessed the value of pCT and DCE-MRI on solitary pulmonary nodules in terms of peak enhancement values (23). More recently, Bisdas et al. (24) concluded that the two imaging modalities were equally suited to differentiate squamous cell carcinoma using deconvolution-based analysis.

In the present study, differences were noticed between the pharmacokinetic parameters derived from the voxel-by-voxel analysis and the volume-averaged time course-derived parameters. The voxel-based $\mathrm{K}^{\text {trans }}$ of $\mathrm{pCT}$ compared largely to those of DCE-MRI. However, the estimation by the tumor volume average-based analysis yielded higher values for DCE-MRI. One explanation could be, from a micromorphologic and physiologic point of view, that tumor tissue is strongly heterogeneous by nature. Moreover, the ability to detect both hypervascular regions (related to angiogenesis) and hypovascular regions (potentially related to hypoxia) is very important. This means that adequate assessment of the tumor tissue requires spatially resolved methods that reflect the 
Table 3. CT and MRI characteristics for perfusion imaging

\begin{tabular}{|c|c|c|}
\hline Parameter & CT & MRI \\
\hline FH coverage & Small $(<3 \mathrm{~cm})$ & Large $(\geq 8 \mathrm{~cm})$ \\
\hline Image orientation & Transverse & Free, can be angulated perpendicular to rectum \\
\hline Time resolution & $\begin{array}{l}\leq 1 \mathrm{~s} \text {, allowing high temporal acquisition of } \\
\text { first pass of tracer }\end{array}$ & $>3 \mathrm{~s}$ \\
\hline Duration perfusion scan & $<2$ min to limit radiation dose and tube heating & $\begin{array}{l}\text { Typically }<12 \text { min, allowing accurate contrast } \\
\text { agent washout acquisition }\end{array}$ \\
\hline Soft-tissue contrast & Low & High on the basis of different relaxation times \\
\hline Contrast agent & Iodinated monomers & Gadolinium chelates \\
\hline Signal-concentration relationship & Linear & Only linear for low concentrations $(<5 \mathrm{mM})$ \\
\hline Safety & Ionizing radiation, effective dose $\sim 15 \mathrm{mSv}$ & None* \\
\hline SNR of dynamic uptake curves & $\begin{array}{l}\text { Typically }<10 \text { (depends on contrast agent } \\
\text { dose and rate) }\end{array}$ & $<50$ (depends on contrast agent dose) \\
\hline Total examination duration & $\sim 5 \mathrm{~min}$ & $\sim 45 \mathrm{~min}$ \\
\hline Availability & $\begin{array}{l}\text { High, frequently also in radiotherapy } \\
\text { departments }\end{array}$ & Low \\
\hline
\end{tabular}

Abbreviations: $\mathrm{SNR}=$ signal-to-noise ratio. Other abbreviations as in Table 1.

* When using particular MR contrast agents there is a very small chance of inducing nephrogenic systemic fibrosis in patients with severe renal impairment.

heterogeneous distribution of microcirculatory properties. Therefore, physiologic properties derived from tumor-averaged uptake measurement seem suboptimal. Another important issue is the higher viscosity of the CT contrast agent compared with the MRI agent $(6.0 \mathrm{vs} .2 .0 \mathrm{mPa} \cdot \mathrm{s})$. Because of the relatively low viscosity of the MRI agent, it can more easily enter tumor tissue regions, both in the capillary system as in the interstitial space. To determine $K^{\text {trans }}, v_{e}$, and $v_{p}$ values, there has to be some (minimal) amount of contrast agent in all tissue compartments, which might not be the case for iodine. Therefore, it is likely that the $v_{e}$ and $v_{p}$ parameter estimates are also influenced by the viscosity of the contrast agent.

Our study is hampered by a few limitations. In general, perfusion measurements are intrinsically variable owing to internal and external factors, including day-to-day physiologic variations (e.g. cardiac output); technical variability; observer variability; tumor heterogeneity (25). Intermodality variations yielding, for example, differences in anatomic coverage; and the relationship between measured signal intensity and concentration of contrast agents. The limited craniocaudal coverage of only $2.88 \mathrm{~cm}$ for $\mathrm{pCT}$ resulted for some patients in an insufficient coverage of the rectal tumor in the imaging volume. For such cases, pCT measurements were only obtained from the region of maximum tumor coverage. Hence, the FOV of DCE-MRI to be analyzed was manually adjusted comparable to the FOV for pCT. The DCE-MRI and pCT measurements could not be performed on the same day, owing to the pharmacokinetic interference of the contrast agents (iodine and gadolinium). However, the interval between both acquisitions was preferred to be within 2 days. A validation with pathology was not feasible owing to the radiother- apy performed between the imaging and the total tumor resection. However, several studies have already compared the pCT- and DCE-MRI-derived angiogenic parameters with histopathologic microcirculation parameters (26-28). Because of differences in patient position and bladder and rectum content, a comparison of the same voxel in the pCT and DCE-MRI scans was very difficult. Accurate image registration between $\mathrm{pCT}$ and DCE-MRI is required but complicated because of these differences. Available nonrigid registration algorithms have a general lack of validation in rectal cancer patients. Therefore, tumor delineation on CT and MRI was performed separately, subsequently assessing the distributions of the pharmacokinetic parameter estimates. In the present study, the delineation of the tumor volume was done by one expert radiation oncologist by using MR and CT images jointly. One might argue that this may introduce some bias with respect to assessments if no MR images were available or studies in which readers are blinded. However, we analyzed whole three-dimensional tumor volumes to avoid comparison of tumor with nontumor tissue. Moreover, the tumors could be easily delineated on both MR and CT images. Assessment of potential delineation differences between MR and CT is outside the scope of the present study, because we wanted to be sure that the same tumor tissue regions were assessed for the pharmacokinetic analysis on MR and CT images.

In conclusion, our study demonstrates for the first time that both pCT and DCE-MRI are suitable and comparable techniques for evaluating tumor perfusion parameters in rectal cancer. This was shown in terms of comparisons between tumor average and voxel-byvoxel $K^{\text {trans }}$ values.

\section{REFERENCES}

1. Padhani AR. Functional MRI for anticancer therapy assessment. Eur J Cancer 2002;38:2116-2127.
2. Miles KA. Perfusion CT for the assessment of tumour vascularity: Which protocol? Br J Radiol 2003;76(Spec No 1):S36-S42. 
3. Goh V, Padhani AR, Rasheed S. Functional imaging of colorectal cancer angiogenesis. Lancet Oncol 2007;8:245-255.

4. Zahra MA, Hollingsworth KG, Sala E, et al. Dynamic contrastenhanced MRI as a predictor of tumour response to radiotherapy. Lancet Oncol 2007;8:63-74.

5. Sahani DV, Kalva SP, Hamberg LM, et al. Assessing tumor perfusion and treatment response in rectal cancer with multisection CT: Initial observations. Radiology 2005;234:785-792.

6. de Vries A, Griebel J, Kremser C, et al. Monitoring of tumor microcirculation during fractionated radiation therapy in patients with rectal carcinoma: Preliminary results and implications for therapy. Radiology 2000;217:385-391.

7. Miles KA, Charnsangavej C, Lee FT, et al. Application of CT in the investigation of angiogenesis in oncology. Acad Radiol 2000;7: 840-850.

8. Padhani AR, Dzik-Jurasz A. Perfusion MR imaging of extracranial tumor angiogenesis. Top Magn Reson Imaging 2004;15: 41-57.

9. McDonald DM, Choyke PL. Imaging of angiogenesis: From microscope to clinic. Nat Med 2003;9:713-725.

10. Goh V, Halligan S, Wellsted DM, et al. Can perfusion CT assessment of primary colorectal adenocarcinoma blood flow at staging predict for subsequent metastatic disease? A pilot study. Eur Radiol 2009;19:79-89.

11. Tofts PS, Brix G, Buckley DL, et al. Estimating kinetic parameters from dynamic contrast-enhanced T(1)-weighted MRI of a diffusable tracer: Standardized quantities and symbols. J Magn Reson Imaging 1999;10:223-232.

12. Pluim JP, Maintz JB, Viergever MA. Mutual-information-based registration of medical images: A survey. IEEE Trans Med Imaging 2003;22:986-1004.

13. Ollers M, Bosmans G, van Baardwijk A, et al. The integration of PET-CT scans from different hospitals into radiotherapy treatment planning. Radiother Oncol 2008;87:142-146.

14. Daisne JF, Sibomana M, Bol A, et al. Tri-dimensional automatic segmentation of PET volumes based on measured source-to-background ratios: Influence of reconstruction algorithms. Radiother Oncol 2003;69:247-250.

15. Heilmann M, Kiessling F, Enderlin M, et al. Determination of pharmacokinetic parameters in DCE MRI: Consequence of nonlinearity between contrast agent concentration and signal intensity. Invest Radiol 2006;41:536-543.

16. Lu H, Clingman C, Golay X, et al. Determining the longitudinal relaxation time (T1) of blood at 3.0 Tesla. Magn Reson Med 2004;52:679-682.
17. Daldrup H, Shames DM, Wendland M, et al. Correlation of dynamic contrast-enhanced magnetic resonance imaging with histologic tumor grade: Comparison of macromolecular and small-molecular contrast media. Pediatr Radiol 1998;28:67-78.

18. Harrer JU, Parker GJ, Haroon HA, et al. Comparative study of methods for determining vascular permeability and blood volume in human gliomas. J Magn Reson Imaging 2004;20: 748-757.

19. Jackson A, Jayson GC, Li KL, et al. Reproducibility of quantitative dynamic contrast-enhanced MRI in newly presenting glioma. Br J Radiol 2003;76:153-162.

20. Walker-Samuel S, Leach MO, Collins DJ. Reference tissue quantification of DCE-MRI data without a contrast agent calibration. Phys Med Biol 2007;52:589-601.

21. Henderson E, Rutt BK, Lee TY. Temporal sampling requirements for the tracer kinetics modeling of breast disease. Magn Reson Imaging 1998;16:1057-1073.

22. Aerts HJ, van Riel NA, Backes WH. System identification theory in pharmacokinetic modeling of dynamic contrast-enhanced MRI: Influence of contrast injection. Magn Reson Med 2008;59: 1111-1119.

23. Kim JH, Kim HJ, Lee KH, et al. Solitary pulmonary nodules: A comparative study evaluated with contrast-enhanced dynamic MR imaging and CT. J Comput Assist Tomogr 2004;28:766-775.

24. Bisdas S, Medov L, Baghi M, et al. A comparison of tumour perfusion assessed by deconvolution-based analysis of dynamic contrast-enhanced CT and MR imaging in patients with squamous cell carcinoma of the upper aerodigestive tract. Eur Radiol 2008;18:843-850.

25. Padhani AR, Hayes C, Landau S, et al. Reproducibility of quantitative dynamic MRI of normal human tissues. NMR Biomed 2002; 15:143-153.

26. de Lussanet QG, Backes WH, Griffioen AW, et al. Dynamic contrast-enhanced magnetic resonance imaging of radiation therapy-induced microcirculation changes in rectal cancer. Int J Radiat Oncol Biol Phys 2005;63:1309-1315.

27. Bellomi M, Petralia G, Sonzogni A, et al. CT perfusion for the monitoring of neoadjuvant chemotherapy and radiation therapy in rectal carcinoma: Initial experience. Radiology 2007;244: 486-493.

28. Zhang XM, Yu D, Zhang HL, et al. 3D dynamic contrast-enhanced MRI of rectal carcinoma at 3T: Correlation with microvascular density and vascular endothelial growth factor markers of tumor angiogenesis. J Magn Reson Imaging 2008;27: 1309-1316. 